\title{
Wavelet Optimal Estimations for Density Functions under Severely Ill-Posed Noises
}

\author{
Rui Li and Youming Liu \\ Department of Applied Mathematics, Beijing University of Technology, Pingle Yuan 100, \\ Beijing 100124, China
}

Correspondence should be addressed to Youming Liu; liuym@bjut.edu.cn

Received 6 October 2013; Accepted 13 November 2013

Academic Editor: Ding-Xuan Zhou

Copyright (c) 2013 R. Li and Y. Liu. This is an open access article distributed under the Creative Commons Attribution License, which permits unrestricted use, distribution, and reproduction in any medium, provided the original work is properly cited.

Motivated by Lounici and Nickl's work (2011), this paper considers the problem of estimation of a density $f$ based on an independent and identically distributed sample $Y_{1}, \ldots, Y_{n}$ from $g=f * \varphi$. We show a wavelet optimal estimation for a density (function) over Besov ball $B_{r, q}^{s}(L)$ and $L^{p}$ risk $(1 \leq p<\infty)$ in the presence of severely ill-posed noises. A wavelet linear estimation is firstly presented. Then, we prove a lower bound, which shows our wavelet estimator optimal. In other words, nonlinear wavelet estimations are not needed in that case. It turns out that our results extend some theorems of Pensky and Vidakovic (1999), as well as Fan and Koo (2002).

\section{Introduction and Preliminary}

Wavelets have made great achievements in studying the statistical model $Y=X+\epsilon$, where $X$ stands for real-valued random variable with unknown probability density $f$, and $\epsilon$ denotes an independent random noise (error) with density $\varphi$.

In 1999, Pensky and Vidakovic [1] investigate Meyer wavelet estimation over Sobolev spaces $W_{2}^{s}(\mathbb{R})$ and $L^{2}$ risk under moderately and severely ill-posed noises. Three years later, Fan and Koo [2] extend those works from $W_{2}^{s}(\mathbb{R})$ to Besov spaces $B_{r, q}^{s}(\mathbb{R})(1 \leq r \leq 2)$. It should be pointed out that, by using different method, Lounici and Nickl [3] study wavelet optimal estimation over Besov spaces $B_{\infty, \infty}^{s}(\mathbb{R})$ and $L^{\infty}$ risk under both noises. In [4], we provide a wavelet optimal estimation over $B_{r, q}^{s}(\mathbb{R})$ and $L^{p}$ risk $(1 \leq p<\infty$, $r, q \in[1, \infty])$ under moderately ill-posed noise. This current paper deals with the same problem under the severely illposed noises. It turns out that our result contains some theorems of $[1,2]$ as special cases. Our discussion also shows that nonlinear wavelet estimations are not needed for severely ill-posed noise, which is totally different with moderately illposed case.
Let $\phi$ and $\psi \in L^{2}(\mathbb{R})$ be a scaling and mother wavelet function, respectively. Then each $f \in L^{2}(\mathbb{R})$ has an expansion (in $L^{2}$ sense):

$$
f=\sum_{k \in \mathbb{Z}} \alpha_{J k} \phi_{J k}+\sum_{j \geq I} \sum_{k \in \mathbb{Z}} \beta_{j k} \psi_{j k}
$$

with $\alpha_{j k}:=\left\langle f, \phi_{j k}\right\rangle$ and $\beta_{j k}:=\left\langle f, \psi_{j k}\right\rangle$. Here and throughout, we use the standard notation $h_{j k}(x):=2^{j / 2} h\left(2^{j} x-k\right)$ in wavelet analysis [5]. A class of important wavelets are Meyer's, whose Fourier transforms are $C^{\infty}$ and compactly supported on $\{t: 2 \pi / 3 \leq|t| \leq 8 \pi / 3\}$ [5]. It is easy to see that $\forall a \geq 0$, $\exists C_{a}>0$ such that $|x|^{a}|\phi(x)| \leq C_{a}$. In this paper, the Fourier transform $\widehat{f}$ for $f \in L(\mathbb{R})$ is defined by

$$
\widehat{f}(t):=(F f)(t)=\int_{\mathbb{R}} f(x) e^{-i t x} d x .
$$

The classical method extends that definition to $L^{2}(\mathbb{R})$ functions. 
The following two lemmas are fundamental in our discussions. We use $\|f\|_{p}$ to denote $L^{p}(\mathbb{R})$ norm of $f \in L^{p}(\mathbb{R})$, and $\|\lambda\|_{p}$ do $l^{p}(\mathbb{Z})$ norm of $\lambda \in l^{p}(\mathbb{Z})$, where

$$
l^{p}(\mathbb{Z}):= \begin{cases}\left\{\lambda=\left\{\lambda_{k}\right\}, \sum_{k \in \mathbb{Z}}\left|\lambda_{k}\right|^{p}<\infty\right\}, & 1 \leq p<\infty, \\ \left\{\lambda=\left\{\lambda_{k}\right\}, \sup _{k \in \mathbb{Z}}\left|\lambda_{k}\right|<\infty\right\}, & p=\infty .\end{cases}
$$

Lemma 1 (see [6]). Let $h$ be a scaling or a wavelet function with $\sup _{x \in \mathbb{R}} \sum_{k \in \mathbb{Z}}|h(x-k)|<\infty$. Then, there exist $C_{2} \geq C_{1}>$ 0 such that for $\lambda=\left\{\lambda_{k}\right\} \in l^{p}(\mathbb{Z})$ with $1 \leq p \leq \infty$,

$$
C_{1} 2^{j(1 / 2-1 / p)}\|\lambda\|_{p} \leq\left\|\sum_{k \in \mathbb{Z}} \lambda_{k} h_{j k}\right\|_{p} \leq C_{2} 2^{j(1 / 2-1 / p)}\|\lambda\|_{p} .
$$

One of the advantages of wavelet bases is that they can characterize Besov spaces. To introduce those spaces [6], we need the well-known Sobolev spaces with integer exponents

$$
W_{p}^{m}(\mathbb{R}):=\left\{f \in L^{p}(\mathbb{R}), f^{(m)} \in L^{p}(\mathbb{R})\right\},
$$

with the Sobolev norm $\|f\|_{W_{p}^{m}}:=\|f\|_{p}+\left\|f^{(m)}\right\|_{p}$. Then $L^{p}(\mathbb{R})$ can be considered as $W_{p}^{(0)}(\mathbb{R})$. For $1 \leq p, q \leq \infty$ and $s=m+\alpha$ with $\alpha \in(0,1]$, a Besov space is defined by

$$
B_{r, q}^{s}(\mathbb{R}):=\left\{f \in W_{r}^{m}(\mathbb{R}),\left\|t^{-\alpha} \omega_{r}^{2}\left(f^{(m)}, t\right)\right\|_{q}^{*}<\infty\right\},
$$

with the norm $\|f\|_{s r q}:=\|f\|_{W_{r}^{m}}+\left\|t^{-\alpha} \omega_{r}^{2}\left(f^{(m)}, t\right)\right\|_{q}^{*}$, where $\omega_{r}^{2}(f, t):=\sup _{|h| \leq t}\|f(\cdot+2 h)-2 f(\cdot+h)+f(\cdot)\|_{r}$ denotes the smoothness modulus of $f$ and

$$
\|h\|_{q}^{*}= \begin{cases}\left(\int_{0}^{\infty}\left(\frac{|h(t)|^{q}}{t}\right) d t\right)^{1 / q}, & \text { if } 1 \leq q<\infty, \\ \operatorname{ess} \sup _{t \in \mathbb{R}}|h(t)|, & \text { if } q=\infty .\end{cases}
$$

Lemma 2 (see [6]). Let $\phi$ be a Meyer scaling function and $\psi$ be the corresponding wavelet. If $f \in L^{r}(\mathbb{R}), 1 \leq r \leq \infty$, $\alpha_{0 k}=\int f(x) \phi_{0 k}(x) d x$, and $\beta_{j k}=\int f(x) \psi_{j k}(x) d x$, then the following assertions are equivalent:

(i) $f \in B_{r, q}^{s}(\mathbb{R}), 1 \leq q \leq \infty$;

(ii) $\left\{2^{j s}\left\|P_{j} f-f\right\|_{r}\right\}_{j \geq 0} \in l^{q}$, where $P_{j} f:=\sum_{k \in \mathbb{Z}} \alpha_{j k} \phi_{j k}$;

(iii) $\left\|\alpha_{0 k}\right\|_{r}+\left\|\left\{2^{j(s+1 / 2-1 / r)}\left\|\beta_{j}\right\|_{r}\right\}_{j \geq 0}\right\|_{q}<\infty$.

In each case,

$$
\|f\|_{s r q} \sim\left\|\alpha_{0} \cdot\right\|_{r}+\left\|\left\{2^{j(s+1 / 2-1 / r)}\left\|\beta_{j} \cdot\right\|_{r}\right\}_{j \geq 0}\right\|_{q} .
$$

Here and after, $A \leqslant B$ denotes $A \leq C B$ for some constant $C>0 ; A \gtrsim B$ means $B \lesssim A ; A \sim B$ stands for both $A \lesssim B$ and $B \lesssim A, \alpha_{0}$. does for the sequence of $\left\{\alpha_{0 k}, k \in \mathbb{Z}\right\}$.
At the end of this subsection, we make some assumptions on noise density $\varphi$, which will be dealt with in this current paper. For $\alpha>0, c>0, \beta \in \mathbb{R}$,

$$
\begin{aligned}
& \text { (C1) }|\widehat{\varphi}(t)| \leq C\left(1+|t|^{2}\right)^{-\beta / 2} e^{-c|t|^{\alpha}} \text { (a.e.); } \\
& \text { (C2) }\left|\widehat{\varphi}^{\prime}(t)\right| \leq C\left(1+|t|^{2}\right)^{-\beta / 2} e^{-c|t|^{\alpha}} \text { (a.e.); } \\
& \text { (C3) }|\widehat{\varphi}(t)| \geq C\left(1+|t|^{2}\right)^{-\beta / 2} e^{-c|t|^{\alpha}} \text { (a.e.). }
\end{aligned}
$$

Clearly, the classical Cauchy densities satisfy (C1)-(C3) with $\alpha=c=1$ and $\beta=0$, and the Gaussian density does satisfy (C1) $-(\mathrm{C} 3)$ with $\alpha=2, c=1 / 2$, and $\beta=0$. It should be pointed out that those above conditions (C1)-(C3) are a little different with [2].

In the next section, we define a wavelet linear estimator and provide an upper bound estimation over Besov spaces $B_{r, q}^{\mathcal{S}}(\mathbb{R})$ and $L^{p}$ risk under the condition (C3); the third part gives a lower bound estimation which shows the result of Section 2 optimal; some concluding remarks are discussed in the last part.

\section{Upper Bound}

To introduce the main theorem of this section, we assume that $Y_{1}, Y_{2}, \ldots, Y_{n}$ are independent and identically distributed (i.i.d) random variables of $Y=X+\epsilon$, the density $\varphi$ of random noise $\epsilon$ satisfies condition (C3), and $\phi$ stands for the Meyer scaling function. As in [1], define

$$
\begin{aligned}
\left(\mathbf{K}_{j} \phi\right)(y) & :=\frac{1}{2 \pi} \int_{\mathbb{R}} e^{i t y} \frac{\widehat{\phi}(t)}{\widehat{\varphi}\left(-2^{j} t\right)} d t, \\
\widehat{\alpha}_{j k} & :=\frac{1}{n} \sum_{i=1}^{n}\left(\mathbf{K}_{j} \phi\right)_{j k}\left(Y_{i}\right),
\end{aligned}
$$

as well as a linear wavelet estimator

$$
f_{n}^{L}:=\sum_{|k| \leq K_{n}} \widehat{\alpha}_{j k} \phi_{j k}
$$

(the positive integer $K_{n}$ will be given later on). Then $\widehat{\alpha}_{j} \in l^{\infty}$, $f_{n}^{L}$ is well defined, and $E f_{n}^{L}=\sum_{|k| \leq K_{n}} \alpha_{j k} \phi_{j k}$.

We use supp $f$ to stand for the support of $f$ and $\mid$ supp $f \mid$ to do its length. Moreover, for $L>0$, denote

$$
\begin{aligned}
& B_{r, q}^{s}(L) \\
& \quad:=\left\{f \in B_{r, q}^{s}(\mathbb{R}), f \geq 0, \int_{\mathbb{R}} f(x) d x=1 \text { and }\|f\|_{s r q} \leq L\right\}, \\
& B_{r, q}^{s}(L, M)=\left\{f \in B_{r, q}^{s}(L),|\operatorname{supp} f| \leq M \text { for some } M>0\right\} .
\end{aligned}
$$

It is reasonable to assume $L>1$ for $r=1$, since $\|f\|_{s 1 q} \geq$ $\|f\|_{1}=1$ in that case. 
Theorem 3. Let $\varphi$ satisfy (C3) and $\phi$ be the Meyer scaling function. If $p \in[1, \infty), q, r \in[1, \infty],\left\|x^{2} f\right\|_{\mu} \leq A(\mu \geq 1, A>$ $0)$, then, with $K_{n} \sim e^{(\ln n)^{\theta}}(0<\theta<1),(3 / 8 \pi)((\ln n) / 4 c)^{1 / \alpha}<$ $2^{j} \leq(3 / 4 \pi)((\ln n) / 4 c)^{1 / \alpha}, s^{\prime}:=s-(1 / r-1 / p)_{+}$, and $x_{+}=$ $\max \{x, 0\}$,

$$
\sup _{f \in B_{r, q}^{s}(L, M)} E\left\|f_{n}^{L}-f\right\|_{p}^{p} \leqslant(\ln n)^{-\left(s^{\prime} / \alpha\right) p} .
$$

In particular, $f \in B_{r, q}^{s}(L, M)$ can be replaced by $f \in B_{r, q}^{s}(L)$, when $r \leq p$.

Proof. When $r \leq p, s^{\prime}:=s-(1 / r-1 / p)_{+}=s-1 / r+1 / p$. Since $l^{r}$ is continuously embedded to $l^{p}$, Lemma 2 implies $B_{r, q}^{s}(\mathbb{R}) \subseteq$ $B_{p, q}^{s^{\prime}}(\mathbb{R})$. Hence,

$$
\sup _{f \in B_{r, q}^{s}(L)} E\left\|f_{n}^{L}-f\right\|_{p}^{p} \lesssim \sup _{f \in B_{p, q}^{\prime}(L)} E\left\|f_{n}^{L}-f\right\|_{p}^{p} .
$$

When $r>p$, one obtains that, for some $C>0, B_{r, q}^{s}(L, M) \subseteq$ $B_{p, q}^{s}(C L, M)$ and

$$
\sup _{f \in B_{r, q}^{s}(L, M)} E\left\|f_{n}^{L}-f\right\|_{p}^{p} \leqslant \sup _{f \in B_{p, q}^{s}(C L, M)} E\left\|f_{n}^{L}-f\right\|_{p}^{p} .
$$

In fact, $f \in B_{r, q}^{s}(L, M)$ and Hölder inequality imply that $\|f\|_{p}^{p}=\int|f(x)|^{p} d x \leqslant\|f(x)\|_{r}^{p}$ due to $|\operatorname{supp} f| \leq M$. By the definition of Besov norm, $\|f\|_{s p q} \leq C\|f\|_{s r q}$. According to (13) and (14), it is sufficient to prove

$$
\sup _{f \in B_{p, q}^{s}(L)} E\left\|f_{n}^{L}-f\right\|_{p}^{p} \lesssim(\ln n)^{-(s / \alpha) p},
$$

for the conclusion of Theorem 3 . Then

Recall that $f_{n}^{L}=\sum_{|k| \leq K_{n}} \widehat{\alpha}_{j k} \phi_{j k}$ and $E f_{n}^{L}=\sum_{|k| \leq K_{n}} \alpha_{j k} \phi_{j k}$.

$$
E\left\|f_{n}^{L}-f\right\|_{p}^{p} \leq E\left\|f_{n}^{L}-E f_{n}^{L}\right\|_{p}^{p}+\left\|\sum_{|k|>K_{n}} \alpha_{j k} \phi_{j k}\right\|_{p}^{p}+\left\|P_{j} f-f\right\|_{p}^{p} .
$$

By $f \in B_{p, q}^{s}(L)$ and Lemma 2 ,

$$
\left\|P_{j} f-f\right\|_{p} \lesssim 2^{-j s} .
$$

To estimate the middle term of (16), one observes that $\alpha_{j k}=\int_{\mathbb{R}} \phi_{j k}(x) f(x) d x,\left|k^{2} \alpha_{j k}\right| \leq \int_{\mathbb{R}} \mid 2^{j} x-k-$ $\left.2^{j} x\right|^{2}\left|\phi_{j k}(x)\right| f(x) d x \leqslant \int_{\mathbb{R}}\left|2^{j} x-k\right|^{2}\left|\phi_{j k}(x)\right| f(x) d x+$ $\int_{\mathbb{R}}\left|2^{j} x\right|^{2}\left|\phi_{j k}(x)\right| f(x) d x$. Since $\phi$ is the Meyer scaling function, $\sup _{x \in \mathbb{R}}|x|^{2}|\phi(x)|<\infty$ and

$$
\int_{\mathbb{R}}\left|2^{j} x-k\right|^{2}\left|\phi_{j k}(x)\right| f(x) d x \leq 2^{j / 2} \sup _{x \in \mathbb{R}}|x|^{2}|\phi(x)| .
$$

On the other hand, $\int_{\mathbb{R}}\left|2^{j} x\right|^{2}\left|\phi_{j k}(x)\right| f(x) d x \leq 2^{2 j}\left\|x^{2} f\right\|_{\mu} \times$ $\left\|\phi_{j k}\right\|_{\mu^{\prime}} \leqslant 2^{j\left(5 / 2-1 / \mu^{\prime}\right)}\left\|x^{2} f\right\|_{\mu}$ with $1 / \mu+1 / \mu^{\prime}=1$. Therefore $\left|k^{2} \alpha_{j k}\right| \lesssim 2^{j / 2}+2^{j\left(5 / 2-1 / \mu^{\prime}\right)} \lesssim 2^{j(5 / 2)}$ and $\sum_{|k|>K_{n}}\left|\alpha_{j k}\right|^{p} \lesssim$ $\sum_{\text {to }}{ }_{|k|>K_{n}} k^{-2 p} 2^{j(5 / 2) p} \lesssim K_{n}^{1-2 p} 2^{j(5 / 2) p}$. This with Lemma 1 leads

$$
\left\|\sum_{|k|>K_{n}} \alpha_{j k} \phi_{j k}\right\|_{p}^{p} \lesssim 2^{j(p / 2-1)} \sum_{|k|>K_{n}}\left|\alpha_{j k}\right|^{p} \leq 2^{j(3 p-1)} K_{n}^{1-2 p} .
$$

Now, it remains to consider $E\left\|f_{n}^{L}-E f_{n}^{L}\right\|_{p}^{p}$ : Using $f_{n}^{L}-$ $E f_{n}^{L}=\sum_{|k| \leq K_{n}}\left(\widehat{\alpha}_{j k}-\alpha_{j k}\right) \phi_{j k}$ and Lemma 1, one knows

$$
E\left\|f_{n}^{L}-E f_{n}^{L}\right\|_{p}^{p} \lesssim 2^{j(p / 2-1)} \sum_{|k| \leq K_{n}} E\left|\widehat{\alpha}_{j k}-\alpha_{j k}\right|^{p} .
$$

Clearly, $\widehat{\alpha}_{j k}-\alpha_{j k}=(1 / n) \sum_{i=1}^{n}\left[\left(\mathbf{K}_{j} \phi\right)_{j k}\left(Y_{i}\right)-E\left(\mathbf{K}_{j} \phi\right)_{j k}\left(Y_{i}\right)\right]$. Define $X_{i, k}:=\left(\mathbf{K}_{j} \phi\right)_{j k}\left(Y_{i}\right)-E\left(\mathbf{K}_{j} \phi\right)_{j k}\left(Y_{i}\right)$. Then $E X_{i, k}=$ 0 and $\widehat{\alpha}_{j k}-\alpha_{j k}=(1 / n) \sum_{i=1}^{n} X_{i, k}$. To apply Rosenthal's inequality (Proposition 10.2, [6]), one estimates $\left|X_{i, k}\right|$ and $E\left|X_{i, k}\right|^{p}$ : note that $\left|\left(\mathbf{K}_{j} \phi\right)_{j k}\left(Y_{i}\right)\right|=2^{j / 2} \mid(1 / 2 \pi) \times$ $\int_{\mathbb{R}} e^{i t\left(2^{j} Y_{i}-k\right)}\left(\widehat{\phi}(t) / \widehat{\varphi}\left(-2^{j} t\right)\right) d t \mid \lesssim 2^{j / 2} \int_{\mathbb{R}}\left(1+\left|2^{j} t\right|^{2}\right)^{\beta / 2} e^{c\left|2^{j} t\right|^{\alpha}} \times$ $|\widehat{\phi}(t)| d t \lesssim 2^{j(\beta+1 / 2)} e^{c\left((4 \pi / 3) 2^{j}\right)^{\alpha}}$ due to (C3) and supp $\widehat{\phi} \subseteq$ $[-(4 / 3) \pi,(4 / 3) \pi]$. Then

$$
\begin{gathered}
\left|X_{i, k}\right| \lesssim 2^{j(\beta+1 / 2)} e^{c\left((4 \pi / 3) 2^{j}\right)^{\alpha}}, \\
E\left|X_{i, k}\right|^{p} \lesssim 2^{j(\beta+1 / 2) p} e^{c p\left((4 \pi / 3) 2^{j}\right)^{\alpha}} .
\end{gathered}
$$

Because $X_{i, k}$ are i.i.d, the Rosenthal's inequality tells that

$$
\begin{aligned}
& E\left|\widehat{\alpha}_{j k}-\alpha_{j k}\right|^{p} \\
& \quad \lesssim \begin{cases}n^{-p}\left[n E\left|X_{i, k}\right|^{p}+n^{p / 2}\left(E\left|X_{i, k}\right|^{2}\right)^{p / 2}\right], & p>2, \\
n^{-p / 2}\left(E\left|X_{i, k}\right|^{2}\right)^{p / 2}, & 0<p \leq 2 .\end{cases}
\end{aligned}
$$

This with (21) implies that, for $p \geq 2, E\left|\widehat{\alpha}_{j k}-\alpha_{j k}\right|^{p} \leqslant$ $n^{1-p} 2^{j(\beta+1 / 2) p} e^{c p\left((4 \pi / 3) 2^{j}\right)^{\alpha}} I\{p>2\}+n^{-p / 2} 2^{j(\beta+1 / 2) p} \times$ $e^{c p\left((4 \pi / 3) 2^{j}\right)^{\alpha}} \lesssim n^{-p / 2} 2^{j(\beta+1 / 2) p} e^{c p\left((4 \pi / 3) 2^{j}\right)^{\alpha}}$. Moveover, (20) reduces to

$$
E\left\|f_{n}^{L}-E f_{n}^{L}\right\|_{p}^{p} \leqslant K_{n} n^{-p / 2} 2^{j(\beta+1) p-j} e^{c p\left((4 \pi / 3) 2^{j}\right)^{\alpha}} .
$$

Then it follows from (16)-(19) and (23) that

$$
\begin{aligned}
E\left\|f_{n}^{L}-f\right\|_{p}^{p} \leq & 2^{-j p s}+2^{j(3 p-1)} K_{n}^{1-2 p} \\
& +K_{n} n^{-p / 2} 2^{j(\beta+1) p-j} e^{c p\left((4 \pi / 3) 2^{j}\right)^{\alpha}} .
\end{aligned}
$$


By the choices $K_{n} \sim e^{(\ln n)^{\theta}}$ and $(3 / 8 \pi)((\ln n) / 4 c)^{1 / \alpha}<2^{j} \leq$ $(3 / 4 \pi)((\ln n) / 4 c)^{1 / \alpha}$ (stated in Theorem 3$)$, one receives that $2^{-j p s} \lesssim(\ln n)^{-(s / \alpha) p}$,

$$
\begin{aligned}
& 2^{j(3 p-1)} K_{n}^{1-2 p} \\
& \quad \lesssim(\ln n)^{(3 p-1) / \alpha} e^{-(2 p-1)(\ln n)^{\theta}} \\
& \quad=o\left((\ln n)^{-(s / \alpha) p}\right), \\
& K_{n} n^{-p / 2} 2^{j(\beta+1) p-j} e^{c p\left((4 \pi / 3) 2^{j}\right)^{\alpha}} \\
& \quad \lesssim e^{(\ln n)^{\theta}} n^{-p / 2}(\ln n)^{((\beta+1) p-1) / \alpha} n^{p / 4} \\
& \quad=o\left((\ln n)^{-(s / \alpha) p}\right) .
\end{aligned}
$$

Finally, the desired conclusion (15) follows.

Remark 4. Note that the choices of $j$ and $K_{n}$ do not depend on the unknown parameters $s, r$, and $q$. Then our linear wavelet estimator $f_{n}^{L}$ over Besov space $B_{r, q}^{s}$ is adaptive or implementable. The same conclusion holds for $L^{\infty}$ and $L^{2}$ estimations; see Theorem 2 in [3] and Corollary 1 in [1]. On the other hand, when $p=2$ and $1 \leq r \leq 2$, our Theorem 3 reduces to Theorem 4 in [2]; from the proof of Theorem 3, we find that, for $p>1$, the assumption $\left\|x^{2} f(x)\right\|_{\mu} \leq A$ can be replaced by $\|x f(x)\|_{\infty} \leq A$, which is the same as in [1]. Therefore, for $p=r=q=2$, Theorem 3 of [1] follows directly from our Theorem 3 .

\section{Lower Bound}

In this part, we will provide a lower bound estimation, which shows Theorem 3 to be the best possible in some sense. The following lemmas are needed in the proof of our main theorem of this section.

Lemma 5. Let $h_{\eta}(x):=\eta p(\eta x)$ with $p(x)=1 / \pi\left(1+x^{2}\right)$, $\eta>0$, and $r, q \geq 1$. Then for $L>0$ ( $L>2$ when $r=1)$, there exists $\eta_{0}>0$ such that $h_{0}:=h_{\eta_{0}} \in B_{r, q}^{s}(L / 2)$. If $\psi$ is the Meyer wavelet function and $\left|\lambda_{k}\right| \leq d 2^{-j / 2}\left(k=1,2, \ldots, 2^{j}\right)$, then, for some small $d>0$,

$$
\left|\sum_{k=1}^{2^{j}} \lambda_{k} \psi_{j k}(x)\right| \leq h_{0}(x) .
$$

Proof. It is easy to see that $\left(1+x^{2}\right)^{-1} \in W_{r}^{m}(\mathbb{R})$ (for $r \geq 1$ ) and $h_{\eta} \in B_{r, q}^{s}(\mathbb{R})(s<m)$ by the definition of Besov space. Since

$$
\begin{aligned}
\left\|h_{\eta}\right\|_{s r q}= & \eta^{1-1 / r}\|p\|_{r}+\eta^{1+\lfloor s\rfloor-1 / r}\left\|p^{(\lfloor s\rfloor)}\right\|_{r} \\
& +\eta^{1+s-1 / r}\left\|\frac{\omega_{r}^{2}\left(p^{(\lfloor s\rfloor)}, t\right)}{t^{s-\lfloor s\rfloor}}\right\|_{q}^{*},
\end{aligned}
$$

where $\lfloor s\rfloor$ denotes the largest integer no more than $s,\left\|h_{0}\right\|_{s r q}$ can be made small enough by choosing small $\eta_{0}>0$, when $r>1$. Clearly, $L>2$ is needed, when $r=1$.

$$
\text { If }\left|\lambda_{k}\right| \leq d 2^{-j / 2}\left(k=1,2, \ldots, 2^{j}\right) \text {, then }\left|\sum_{k=1}^{2^{j}} \lambda_{k} \psi_{j k}(x)\right| \leq
$$
$d \sum_{k=1}^{2^{j}}\left|\psi\left(2^{j} x-k\right)\right| \lesssim d \sum_{k=1}^{2^{j}}\left(1+\left|2^{j} x-k\right|^{2}\right)^{-1}$ because $\psi$ is the Meyer function. Note that $\sum_{k=1}^{2^{j}}\left(1+\left|2^{j} x-k\right|^{2}\right)^{-1}=\sum_{k=1}^{2^{j}}(1+$ $\left.2^{2 j}\left|x-2^{-j} k\right|^{2}\right)^{-1} \leq 2^{-2 j} \sum_{k=1}^{2^{j}}\left(|x|^{2}-1\right)^{-1}=2^{-j}\left(x^{2}-1\right)^{-1}$. Then for some small $d>0$ and $|x| \geq 2$,

$$
\left|\sum_{k=1}^{2^{j}} \lambda_{k} \psi_{j k}(x)\right| \leq 2^{-j} h_{0}(x)
$$

Hence, (26) holds for $|x| \geq 2$. On the other hand, when $|x|<$ $2, h_{0}(x) \gtrsim 1$ and $\left|\sum_{k=1}^{2^{j}} \lambda_{k} \psi_{j k}(x)\right| \leq d \sum_{k=1}^{2^{j}}\left|\psi\left(2^{j} x-k\right)\right| \leq$ $d \sup _{x} \sum_{k \in \mathbb{Z}}|\psi(x-k)| \leq C d \leq h_{0}(x)$. Therefore, (26) is true, when $d>0$ small enough. This completes the proof of Lemma 5.

The next lemma extends an estimate in the proof of Theorem 1 in [3].

Lemma 6. Let $\psi$ be the Meyer wavelet function, $h_{0}(x)$, defined as in Lemma 5. If $\varphi$ satisfies (C1), (C2), and $\omega_{k} \in\{0,1\}$, then

$$
\begin{gathered}
\int_{\mathbb{R}}\left(h_{0} * \varphi\right)^{-1}(y)\left(\sum_{k=1}^{2^{j}} \omega_{k} \psi_{j k} * \varphi\right)^{2}(y) d y \\
\lesssim 2^{-2 j \beta} e^{-2 c\left((2 \pi / 3) 2^{j}\right)^{\alpha}} \sum_{k=1}^{2^{j}} \omega_{k}^{2} .
\end{gathered}
$$

Proof. As shown in proof of Theorem 1 of [3], one finds easily that $\left(h_{0} * \varphi\right)(y) \gtrsim\left(1+y^{2}\right)^{-1}$ and therefore

$$
\begin{gathered}
\int_{\mathbb{R}}\left(h_{0} * \varphi\right)^{-1}(y)\left(\sum_{k=1}^{2^{j}} \omega_{k} \psi_{j k} * \varphi\right)^{2}(y) d y \\
\quad \lesssim \int_{\mathbb{R}}\left(1+y^{2}\right)\left(\sum_{k=1}^{2^{j}} \omega_{k} \psi_{j k} * \varphi\right)^{2}(y) d y .
\end{gathered}
$$

By Parseval identity, (C1) and supp $\widehat{\psi_{j k}} \subseteq\{y,|y| \geq(2 \pi /$ $\left.3) 2^{j}\right\}, \int_{\mathbb{R}}\left(\sum_{k=1}^{2^{j}} \omega_{k} \psi_{j k} * \varphi\right)^{2}(y) d y=(1 / 2 \pi) \int_{|t| \geq(2 \pi / 3) 2^{j}} \times$ $\left|\sum_{k=1}^{2^{j}} \omega_{k} \widehat{\psi_{j k}}(t)\right|^{2}|\widehat{\varphi}(t)|^{2} d t \lesssim 2^{-2 \beta j} e^{-2 c\left((2 \pi / 3) 2^{j}\right)^{\alpha}} \int_{|t| \geq(2 \pi / 3) 2^{j}} \times$ $\left|\sum_{k=1}^{2^{j}} \omega_{k} \widehat{\psi_{j k}}(t)\right|^{2} d t$. Moreover, the orthonormality of $\widehat{\psi_{j k}}$ concludes that

$$
\int_{\mathbb{R}}\left(\sum_{k=1}^{2^{j}} \omega_{k} \psi_{j k} * \varphi\right)^{2}(y) d y \lesssim C 2^{-2 \beta j} e^{-2 c\left((2 \pi / 3) 2^{j}\right)^{\alpha}} \sum_{k=1}^{2^{j}} \omega_{k}^{2} .
$$

To estimate $\int_{\mathbb{R}} y^{2}\left(\sum_{k=1}^{2^{j}} \omega_{k} \psi_{j k} * \varphi\right)^{2}(y) d y$, one proves an inequality:

$$
I:=\int_{\mathbb{R}}\left|\sum_{k=1}^{2^{j}} \omega_{k} \frac{d}{d t} \widehat{\psi_{j k}}(t)\right|^{2} d t \leq C \sum_{k=1}^{2^{j}} \omega_{k}^{2}
$$


Note that $\sum_{k=1}^{2^{j}} \omega_{k} \psi_{0 k}(x), x \sum_{k=1}^{2^{j}} \omega_{k} \psi_{0 k}(x) \in L(\mathbb{R})$, and $\sum_{k=1}^{2^{j}} \omega_{k}(d / d t) \widehat{\psi_{j k}}(t)=2^{-(3 / 2) j}\left[(d / d t) F\left(\sum_{k=1}^{2^{j}} \omega_{k} \psi_{0 k}\right)\right]\left(2^{-j} t\right)$. Then $\int_{\mathbb{R}}\left[(d / d t) F\left(\sum_{k=1}^{2^{j}} \omega_{k} \psi_{0 k}\right)\right]^{2}(t) d t=\int_{\mathbb{R}}|i x|^{2} \mid \sum_{k=1}^{2^{j}} \omega_{k} \times$ $\left.\psi_{0 k}(x)\right|^{2} d x=\sum_{k=1}^{2^{j}} \sum_{k^{\prime}=1}^{2^{j}} \omega_{k} \omega_{k^{\prime}} \int_{\mathbb{R}}(x+k)^{2} \psi(x) \psi\left[x-\left(k^{\prime}-\right.\right.$ $k)] d x$ and

$$
I=2^{-2 j} \sum_{k=1}^{2^{j}} \sum_{k^{\prime}=1}^{2^{j}} \omega_{k} \omega_{k^{\prime}} \int_{\mathbb{R}}(x+k)^{2} \psi(x) \psi\left[x-\left(k^{\prime}-k\right)\right] d x .
$$

Since $\langle\psi(x), \psi(x-k)\rangle=\delta_{k, 0}, \sum_{k=1}^{2^{j}} \sum_{k^{\prime}=1}^{2^{j}} \omega_{k} \omega_{k^{\prime}}{ }^{2} \int_{\mathbb{R}} \psi(x) \psi(x+$ $\left.k-k^{\prime}\right) d x=\sum_{k=1}^{2^{j}} \omega_{k}^{2} k^{2} \leq 2^{2 j} \sum_{k=1}^{2^{j}} \omega_{k}^{2}$; On the other hand, the boundedness of $\int_{\mathbb{R}} x^{2} \psi(x) \psi(x+l) d x$ and $\int_{\mathbb{R}} x \psi(x) \psi(x+l) d x$ implies that

$$
\begin{gathered}
\left|\sum_{k=1}^{2^{j}} \sum_{k^{\prime}=1}^{2^{j}} \omega_{k} \omega_{k^{\prime}} \int_{\mathbb{R}} x^{2} \psi(x) \psi\left(x+k-k^{\prime}\right) d x\right| \\
\lesssim \sum_{k=1}^{2^{j}} \sum_{k^{\prime}=1}^{2^{j}} \omega_{k} \omega_{k^{\prime}} \lesssim 2^{j} \sum_{k=1}^{2^{j}} \omega_{k}^{2},
\end{gathered}
$$

as well as $\left|\sum_{k=1}^{2^{j}} \sum_{k^{\prime}=1}^{2^{j}} \omega_{k} \omega_{k^{\prime}} k \int_{\mathbb{R}} x \psi(x) \psi\left(x+k-k^{\prime}\right) d x\right| \lesssim$ $2^{2 j} \sum_{k=1}^{2^{j}} \omega_{k}^{2}$. Hence, $I \leqslant \sum_{k=1}^{2^{j}} \omega_{k}^{2}$, which reaches (32).

Define $q(t):=F\left(\sum_{k=1}^{2^{j}} \omega_{k} \psi_{j k} * \varphi\right)(t)$. Then $q, q^{\prime} \in L(\mathbb{R})$ and $q$ is locally absolutely continuous. Therefore, $\widehat{q^{\prime}}(y)=$ $i y \widehat{q}(y)=i y\left(\sum_{k=1}^{2^{j}} \omega_{k} \psi_{j k} * \varphi\right)(-y)$ and

$$
\begin{aligned}
\int_{\mathbb{R}} y^{2}\left(\sum_{k=1}^{2^{j}} \omega_{k} \psi_{j k} * \varphi\right)^{2}(y) d y & =\int_{\mathbb{R}}\left|\widehat{q^{\prime}}(y)\right|^{2} d y \\
& =2 \pi \int_{\mathbb{R}}\left|q^{\prime}(t)\right|^{2} d t .
\end{aligned}
$$

Clearly, $q^{\prime}(t)=\sum_{k=1}^{2^{j}} \omega_{k}\left[\widehat{\psi_{j k}}(t) \widehat{\varphi}^{\prime}(t)+\widehat{\varphi}(t)(d / d t) \widehat{\psi_{j k}}(t)\right]$ and $\int_{\mathbb{R}}\left|q^{\prime}(t)\right|^{2} d t \leq 2\left[\int_{\mathbb{R}}\left|\widehat{\varphi}(t) \sum_{k=1}^{2^{j}} \omega_{k}(d / d t) \widehat{\psi_{j k}}(t)\right|^{2} d t+\int_{\mathbb{R}} \mid \widehat{\varphi}^{\prime}(t) \times\right.$ $\left.\left.\sum_{k=1}^{2^{j}} \omega_{k} \widehat{\psi_{j k}}(t)\right|^{2} d t\right] \lesssim 2^{-2 j \beta} e^{-2 c\left((2 \pi / 3) 2^{j}\right)^{\alpha}}\left[\int_{\mathbb{R}} \mid \sum_{k=1}^{2^{j}} \omega_{k}(d / d t) \times\right.$ $\left.\left.\widehat{\psi_{j k}}(t)\right|^{2} d t+2 \int_{\mathbb{R}}\left|\sum_{k=1}^{2^{j}} \omega_{k} \widehat{\psi_{j k}}(t)\right|^{2} d t\right]$ thanks to (C1), (C2), and

$$
\operatorname{supp} \widehat{\psi_{j k}} \subseteq\left\{y,|y| \geq \frac{2 \pi}{3} 2^{j}\right\} .
$$

Moreover, $\int_{\mathbb{R}}\left|q^{\prime}(t)\right|^{2} d t \leq C 2^{-2 j \beta} e^{-2 c\left((2 \pi / 3) 2^{j}\right)^{\alpha}} \sum_{k=1}^{2^{j}} \omega_{k}^{2}$ because of (32) and the orthonormality of $\psi_{j k}$. This with (35), (31), and (30) leads to the desired conclusion of Lemma 6.

Two more classical theorems play important roles in our discussions. We list the first one as Lemma 7, which can be found in [7].

Lemma 7 (Varshamov-Gilbert). Let $\Omega=\left\{\omega=\left(\omega_{1}, \ldots, \omega_{m}\right)\right.$, $\left.\omega_{k} \in\{0,1\}\right\}$ with $m \geq 8$. Then there exists a subset $\left\{\omega^{(0)}, \ldots, \omega^{(M)}\right\}$ of $\Omega$ such that $M \geq 2^{m / 8}, \omega^{(0)}=(0, \ldots, 0)$, and for $j, l=0,1, \ldots, M, j \neq l$,

$$
\sum_{k=1}^{m}\left|\omega_{k}^{(j)}-\omega_{k}^{(l)}\right| \geq \frac{m}{8}
$$

Given two probability measures $P$ and $Q$ on a measurable space $(\mathbb{X}, \mathscr{F})$, the Kullback divergence of $P$ and $Q$ is defined by

$$
K(P, Q):= \begin{cases}\int \ln \left(\frac{d P}{d Q}\right) d P, & \text { if } P \ll Q, \\ +\infty, & \text { otherwise. }\end{cases}
$$

Here, $P \ll Q$ stands for $P$ absolutely continuous with respect to $Q$. In that case, $K(P, Q)=\int \ln \left(f_{P}(x) / f_{\mathrm{Q}}(x)\right) f_{P}(x) d x$, where the function $f_{P}(x)$ denotes the density function of $P$. The second classical theorem is taken from [8].

Lemma 8 (Fano). Let $\left(\mathbb{X}, \mathscr{F}, P_{k}\right)$ be probability measurable spaces and $A_{k} \in \mathscr{F}, k=0,1, \ldots, m$. If $A_{k} \cap A_{v}=\emptyset$ for $k \neq v$, then

$$
\sup _{0 \leq k \leq m} P_{k}\left(A_{k}^{c}\right) \geq \min \left\{\frac{1}{2}, C_{0} \sqrt{m} e^{-\mathrm{K}_{m}}\right\},
$$

where $\mathrm{K}_{m}:=\inf _{0 \leq v \leq m}(1 / m) \sum_{k \neq v} K\left(P_{k}, P_{v}\right), C_{0}=e^{-3 e^{-1}}$, and $A^{c}$ denotes the complement of a set $A$.

Now, we are in the position to state the main theorem in this section.

Theorem 9. Let $\varphi$ satisfy (C1) and (C2), and let $f_{n}(\cdot):=$ $f_{n}\left(Y_{1}, Y_{2}, \ldots, Y_{n}, \cdot\right)$ be an estimator of $f \in B_{r, q}^{s}(L)$. Then for $s>0, p \in[1, \infty), q, r \in[1, \infty]$, and $s \geq 1 / r$, there exists $C>0$ independent of $f_{n}$ such that with $s^{\prime}:=s-(1 / r-1 / p)_{+}$,

$$
\sup _{f \in B_{r, q}^{s}(L)} E\left\|f_{n}-f\right\|_{p}^{p} \geq C(\ln n)^{-\left(s^{\prime} / \alpha\right) p} .
$$

Proof. Assume that $\psi$ is the Meyer wavelet function, then $\psi_{j k} \in B_{r, q}^{s}(\mathbb{R})$. By Lemma 2 ,

$$
\begin{aligned}
\left\|2^{-j(s+1 / 2)} \sum_{k=1}^{2^{j}} \omega_{k} \psi_{j k}\right\|_{B_{r, q}^{s}} \leq & 2^{-j(s+1 / 2)} 2^{j(s-1 / r+1 / 2)} \\
& \times\left(\sum_{k=1}^{2^{j}}\left|\omega_{k}\right|^{r}\right)^{1 / r} \leq 1,
\end{aligned}
$$

for $\omega_{k} \in\{0,1\}$. Furthermore, with the function $h_{0}$ defined in Lemma 5, there exists $c_{1}>0$ such that $h_{\omega}:=h_{0}+$ $c_{1} 2^{-j(s+1 / 2)} \sum_{k=1}^{2^{j}} \omega_{k} \psi_{j k} \geq 0$ and $\left\|h_{\omega}\right\|_{s r q} \leq L$ due to that Lemma. Define

$$
\Lambda:=\left\{h_{\omega}=h_{0}+c_{1} 2^{-j(s+1 / 2)} \sum_{k=1}^{2^{j}} \omega_{k} \psi_{j k}, \omega_{k} \in\{0,1\}\right\} .
$$


Then $\int h_{\omega}(x) d x=1$ because $\int \psi(x) d x=0$ and $\int h_{0}(x) d x=$ 1 .

By Lemma 7, one finds $\Lambda^{\prime}:=\left\{h_{\omega^{(0)}}, h_{\omega^{(1)}}, \ldots, h_{\omega^{(M)}}\right\} \subseteq \Lambda$ with $M \geq 2^{2^{j} / 8}$ and $h_{\omega^{(0)}}=h_{0}$ such that for $\omega \neq \omega^{\prime}$ and $h_{\omega}$, $h_{\omega^{\prime}} \in \Lambda^{\prime}, \sum_{k=1}^{2^{j}}\left|\omega_{k}-\omega_{k}^{\prime}\right| \geq 2^{j} / 8$. It is easy to see that

$$
\left\|h_{\omega}-h_{\omega^{\prime}}\right\|_{p}=c_{1} 2^{-j(s+1 / 2)}\left\|\sum_{k=1}^{2^{j}}\left(\omega_{k}-\omega_{k}^{\prime}\right) \psi_{j k}\right\|_{p} .
$$

This with Lemma 1 leads to $\left\|h_{\omega}-h_{\omega^{\prime}}\right\|_{p} \gtrsim c_{1} 2^{-j(s+1 / 2)} \times$ $2^{j(1 / 2-1 / p)}\left(\sum_{k=1}^{2^{j}}\left|\omega_{k}-\omega_{k}^{\prime}\right|\right)^{1 / p} \gtrsim c_{1} 2^{-j s}$, and therefore

$$
\theta_{j}:=\min _{h_{\omega} \neq h_{\omega^{\prime}} \in \Lambda^{\prime}}\left\|h_{\omega}-h_{\omega^{\prime}}\right\|_{p} \gtrsim c_{1} 2^{-j s} \text {. }
$$

Define $A_{\omega}:=\left\{\left\|f_{n}-h_{\omega}\right\|_{p}<\theta_{j} / 2\right\}$ for $h_{\omega} \in \Lambda^{\prime}$. Then $A_{\omega} \cap$ $A_{\omega^{\prime}}=\emptyset$, when $\omega \neq \omega^{\prime}$. Clearly, $h_{\omega} * \varphi$ is a density function because both $h_{\omega}$ and $\varphi$ are density functions. Let $P_{h_{\omega} * \varphi}^{n}$ be the probability measure on the Lebesgue space $\left(\mathbb{R}^{n}, \mathscr{L}\right)$ with the density $\prod_{i=1}^{n}\left(h_{\omega} * \varphi\right)\left(y_{i}\right)$. Then Lemma 8 tells that

$$
\sup _{h_{\omega} \in \Lambda^{\prime}} P_{h_{\omega} * \varphi}^{n}\left\{\left\|f_{n}-h_{\omega}\right\|_{p}>\frac{\theta_{j}}{2}\right\} \geq \min \left\{\frac{1}{2}, C_{0} M^{1 / 2} e^{-K_{M}}\right\} .
$$

According to Lemma 5, $h_{\omega}(x) \lesssim h_{0}(x)$ and $P_{h_{\omega} * \varphi}^{n} \ll$ $P_{h_{0} * \varphi}^{n}$. Moreover, $K\left(P_{h_{\omega} * \varphi}^{n}, P_{h_{0} * \varphi}^{n}\right)=\int_{\mathbb{R}^{\star}}\left[\ln \left(\Pi_{i=1}^{n}\left(h_{\omega} * \varphi\right)\left(y_{i}\right) /\right.\right.$ $\left.\left.\prod_{i=1}^{n}\left(h_{0} * \varphi\right)\left(y_{i}\right)\right)\right] \prod_{i=1}^{n}\left(h_{\omega} * \varphi\right)\left(y_{i}\right) d y_{1} d y_{2} \cdots d y_{n}=$ $n \int_{\mathbb{R}} \ln \left(\left(h_{\omega} * \varphi\right)(y) /\left(h_{0} * \varphi\right)(y)\right)\left(h_{\omega} * \varphi\right)(y) d y=n \int_{\mathbb{R}} \ln [1+$ $\left.c_{1} 2^{-j(s+1 / 2)}\left(\sum_{k=1}^{2^{j}} \omega_{k} \psi_{j k} * \varphi\right)\left(h_{0} * \varphi\right)^{-1}\right]\left(h_{\omega} * \varphi\right)(y) d y$. Since $h_{\omega} * \varphi / h_{0} * \varphi>0, c_{1} 2^{-j(s+1 / 2)}\left(\sum_{k=1}^{2^{j}} \omega_{k} \psi_{j k} * \varphi\right)\left(h_{0} * \varphi\right)^{-1}=$ $\left(h_{\omega} * \varphi-h_{0} * \varphi\right) / h_{0} * \varphi>-1$. Combining this with $\ln (1+x) \leq x(x>-1)$, one knows

$$
\begin{aligned}
& K\left(P_{h_{\omega} * \varphi}^{n}, P_{h_{0} * \varphi}^{n}\right) \leq n c_{1} 2^{-j(s+1 / 2)} \\
& \times \int_{\mathbb{R}}\left(h_{0} * \varphi\right)^{-1}(y) \\
& \quad \times\left(\sum_{k=1}^{2^{j}} \omega_{k} \psi_{j k} * \varphi\right)(y) \\
& \times\left(h_{\omega} * \varphi\right)(y) d y .
\end{aligned}
$$

Because $\int_{\mathbb{R}}\left(\psi_{j k} * \varphi\right)(y) d y=F\left(\psi_{j k} * \varphi\right)(0)=\widehat{\psi_{j k}}(0) \widehat{\varphi}(0)=$ 0 , the above inequality reduces to $K\left(P_{h_{\omega} * \varphi}^{n}, P_{h_{0} * \varphi}^{n}\right) \leq$ $c_{1}^{2} n 2^{-2 j(s+1 / 2)} \int\left[\left(\sum_{k=1}^{2^{j}} \omega_{k} \psi_{j k} * \varphi\right)^{2}(y)\right]\left[\left(h_{0} * \varphi\right)(y)\right]^{-1} d y \lesssim$ $c_{1}^{2} n 2^{-2 j(s+1 / 2+\beta)} \exp \left\{-2 c\left((2 \pi / 3) 2^{j}\right)^{\alpha}\right\} \sum_{k=1}^{2^{j}} \omega_{k}^{2} \leq c_{1}^{2} n 2^{-2 j(s+\beta)} \times$ $\exp \left\{-2 c\left((2 \pi / 3) 2^{j}\right)^{\alpha}\right\}$ thanks to Lemma 6. Hence,

$$
\begin{aligned}
\mathrm{K}_{M} & :=\inf _{0 \leq v \leq m} \frac{1}{m} \sum_{k \neq v} K\left(P_{k}, P_{v}\right) \\
& \leq c_{1}^{2} n 2^{-2 j(s+\beta)} \exp \left\{-2 c\left(\frac{2 \pi}{3} 2^{j}\right)^{\alpha}\right\} .
\end{aligned}
$$

Note that $M \geq 2^{2^{j} / 8}$ and take $j$ such that

$$
c^{-1}\left(\frac{2 \pi}{3}\right)^{-\alpha} \ln n<2^{j \alpha} \leq 2^{\alpha} c^{-1}\left(\frac{2 \pi}{3}\right)^{-\alpha} \ln n .
$$

Then $M^{1 / 2} e^{-\mathrm{K}_{M}} \geq \exp \left\{((\ln 2) / 16)(3 / 2 \pi) c^{-1 / \alpha}(\ln n)^{1 / \alpha}\right\}$ $\exp \left\{-C c_{1}^{2} n^{-1}\left[c^{-1 / \alpha}(3 / 2 \pi)(\ln n)^{1 / \alpha}\right]^{-2(s+\beta)}\right\} \gtrsim 1$ (choose $c_{1}>0$ small enough). Furthermore, (45) reduces to

$$
\sup _{h_{\omega} \in \Lambda^{\prime}} P_{h_{\omega} * \varphi}^{n}\left\{\left\|f_{n}-h_{\omega}\right\|_{p}>\frac{\theta_{j}}{2}\right\} \gtrsim 1 .
$$

Hence, $\sup _{f \in B_{r, q}^{s}(L)} E\left\|f_{n}-f\right\|_{p}^{p} \geq \sup _{h_{\omega} \in \Lambda^{\prime}} E\left\|f_{n}-h_{\omega}\right\|_{p}^{p} \geq$ $\sup _{h_{\omega} \in \Lambda^{\prime}} P_{h_{\omega} * \varphi}^{n}\left\{\left\|f_{n}-h_{\omega}\right\|_{p}>\theta_{j} / 2\right\}\left(\theta_{j} / 2\right)^{p} \gtrsim \theta_{j}^{p}$. This with (44) and (48) leads to

$$
\sup _{f \in B_{r, q}^{s}(L)} E\left\|f_{n}-f\right\|_{p}^{p} \gtrsim(\ln n)^{-s p / \alpha},
$$

which is the desired conclusion of Theorem 9, when $r \geq p$ $\left(s^{\prime}=s\right.$ in that case $)$.

When $r<p, s^{\prime}=s-(1 / r-1 / p)_{+}=s-1 / r+1 / p$, it remains to show

$$
\sup _{f \in B_{r, q}^{s}(L)} E\left\|f_{n}-f\right\|_{p}^{p} \geq C(\ln n)^{-((s-1 / r+1 / p) / \alpha) p} .
$$

Similar to the proof of (50), one takes small $c_{2}>0$ such that

$$
h_{k}(x):=h_{0}(x)+c_{2} 2^{-j(s-1 / r+1 / 2)} \psi_{j k}(x) \quad\left(k=1, \ldots, 2^{j}\right)
$$

satisfies $h_{k}(x) \geq 0, h_{k} \in B_{r, q}^{s}(L)$ and $\int h_{k}(x) d x=1$. Clearly, $\left\|h_{k}-h_{0}\right\|_{p}=c_{2} 2^{-j(s-1 / r+1 / 2)} 2^{j(1 / 2-1 / p)}\|\psi\|_{p}$ and $\left\|h_{k}-h_{k^{\prime}}\right\|_{p}=c_{2} 2^{-j(s-1 / r+1 / p)}\left\|\psi(\cdot)-\psi\left(\cdot-\left(k^{\prime}-k\right)\right)\right\|_{p}$ for $1 \leq$ $k \neq k^{\prime} \leq 2^{j}$. Since $\psi$ is the Meyer wavelet function, $\inf _{k \neq 0}\|\psi(\cdot)-\psi(\cdot-k)\|_{p}>0$ and

$$
\delta_{j}:=\min _{0 \leq k \neq k^{\prime} \leq 2^{j}}\left\|h_{k}-h_{k^{\prime}}\right\|_{p} \gtrsim c_{2} 2^{-j(s-1 / r+1 / p)} .
$$

Define $A_{k}:=\left\{\left\|f_{n}-h_{k}\right\|_{p}<\delta_{j} / 2\right\}\left(k=0,1, \ldots, 2^{j}\right)$. Then $A_{k} \cap A_{v}=\emptyset(k \neq v)$ and

$$
\sup _{k=0,1, \ldots, 2^{j}} P_{h_{k} * \varphi}^{n}\left\{\left\|f_{n}-h_{k}\right\|_{p} \geq \frac{\delta_{j}}{2}\right\} \geq \min \left\{\frac{1}{2}, C_{0} 2^{j / 2} e^{-K_{2 j}}\right\},
$$

due to Lemma 8. Similar (even simpler) arguments to the estimation of $\mathrm{K}_{M}$ show $\mathrm{K}_{2^{j}} \lesssim c_{2}^{2} n 2^{-2 j(s-1 / r+\beta+1 / 2)} e^{-2 c\left((2 \pi / 3) 2^{j}\right)^{\alpha}}$. Taking $j$ as in (48), one receives that

$$
\mathrm{K}_{2^{j}} \leq C c_{2}^{2} n^{-1}\left[c^{-1}\left(\frac{2 \pi}{3}\right)^{-\alpha} \ln n\right]^{-(2(s-1 / r+\beta+1 / 2)) / \alpha},
$$

and $2^{j / 2} e^{-K_{2} j} \geq\left[c^{-1}(2 \pi / 3)^{-\alpha} \ln n\right]^{1 / 2 \alpha} \exp \left\{-C c_{2}^{2}\left[c^{-1}(2 \pi /\right.\right.$ $\left.\left.3)^{-\alpha} \ln n\right]^{-(2(s-1 / r+\beta+1 / 2)) / \alpha} n^{-1}\right\} \geq 1$ by choosing small $c_{2}>0$. Thus (54) reduces to

$$
\sup _{k=0,1, \ldots, 2^{j}} P_{h_{k} * \varphi}^{n}\left\{\left\|f_{n}-h_{k}\right\|_{p} \geq \frac{\delta_{j}}{2}\right\} \geq 1 .
$$


Moreover, $\sup _{f \in B_{r, q}^{s}(L)} E_{f * \varphi}^{n}\left\|f_{n}-f\right\|_{p}^{p} \geq \sup _{k=0,1, \ldots, 2^{j}} E_{h_{k} * \varphi}^{n} \| f_{n^{-}}$ $h_{k} \|_{p}^{p} \geq \sup _{k=0,1, \ldots, 2^{j}} P_{h_{k} * \varphi}^{n}\left\{\left\|f_{n}-h_{k}\right\|_{p} \geq \delta_{j} / 2\right\}\left(\delta_{j} / 2\right)^{p} \geq \delta_{j}^{p}$. This with (53) and (48) leads to (51). This completes the proof of Theorem 9.

Remark 10. By Theorems 9 and 3, the linear wavelet estimator $f_{n}^{L}$ is optimal for a density in Besov spaces with severely illposed noise. Therefore, we do not need to consider nonlinear wavelet estimations in that case. This contrasts sharply with moderately ill-posed noise case under which nonlinear wavelet estimation improves the linear one $[2,4]$.

Remark 11. When $p=2$ and $1 \leq r \leq 2$, our Theorem 9 is better than Theorem 6 in [2], because $(\ln n)^{-s^{\prime} / \alpha}>(\ln n)^{-s / \alpha}$. Moreover, Theorems 9 and 3 lead to Theorem 3 in that paper for $p=2$ and $1 \leq r \leq 2$. In addition, our conditions (C1) and (C2) are a little weaker than the assumptions in [2].

\section{Concluding Remarks}

This paper provides an $L^{p}(1 \leq p<\infty)$ risk upper bound for a linear wavelet estimator $f_{n}^{L}$ (Theorem 3 ), which turns out to be optimal (Theorem 9). Therefore, nonlinear estimations are not needed under severely ill-posed noises. Although we assume $p<\infty$ in Theorem 9 , the proof of that theorem shows that, for $p=\infty$,

$$
\sup _{f \in B_{r, q}^{s}(L)} E\left\|f_{n}-f\right\|_{\infty} \geq C(\ln n)^{-(s-1 / r) / \alpha}
$$

In particular, when $r=q=\infty$, this above estimation reduces to partial result of Theorem 1 in [3].

Note that our model assumes the noise to be severely illposed; that is, the density $\varphi$ of noise $\epsilon$ satisfies $|\widehat{\varphi}(t)| \sim(1+$ $\left.|t|^{2}\right)^{-\beta / 2} e^{-c|t|^{\alpha}}$ (a.e.). Then it is reasonable to choose the Meyer scaling function as $\phi$ because the compact supportness of $\widehat{\phi}$ makes $\mathbf{K}_{j} \phi$ well defined, where

$$
\left(\mathbf{K}_{j} \phi\right)(y):=\frac{1}{2 \pi} \int_{\mathbb{R}} e^{i t y} \frac{\widehat{\phi}(t)}{\widehat{\varphi}\left(-2^{j} t\right)} d t
$$

Compare with the proof of Theorem 1 in [3], the arguments of Theorem 9 are more complicated in the sense that we use Varshmov-Gilbert Lemma (Lemma 7). It is reasonable because we deal with unmatched estimation $\sup _{f \in B_{r, q}^{s}(\mathbb{R})} E\left\|f_{n}-f\right\|_{p}^{p}$ ( $p$ and $r$ may not be equal), while they do the matched case $\sup _{f \in B_{\infty, \infty}^{s}(\mathbb{R})} E\left\|f_{n}-f\right\|_{\infty}$.

Although the Shannon function $\phi^{S}(t)=\sin \pi t / \pi t$ is much simpler than the Meyer's, it cannot be used in our discussion because the Shannon's does not belong to $L(\mathbb{R})$, while our theorems cover the case for $p=1$.

Finally, it should be pointed out that we assume the independence of observations $Y_{1}, Y_{2}, \ldots, Y_{n}$ in this paper. However, some dependent data are more important (of course, more complicated) in practice. We will investigate that case in future.

\section{Acknowledgment}

This paper is supported by the National Natural Science Foundation of China (no. 11271038).

\section{References}

[1] M. Pensky and B. Vidakovic, "Adaptive wavelet estimator for nonparametric density deconvolution," The Annals of Statistics, vol. 27, no. 6, pp. 2033-2053, 1999.

[2] J. Fan and J.-Y. Koo, "Wavelet deconvolution," IEEE Transactions on Information Theory, vol. 48, no. 3, pp. 734-747, 2002.

[3] K. Lounici and R. Nickl, "Global uniform risk bounds for wavelet deconvolution estimators," The Annals of Statistics, vol. 39, no. 1, pp. 201-231, 2011.

[4] R. Li and Y. Liu, "Wavelet optimal estimations for a density with some additive noises," Applied and Computational Harmonic Analysis, 2013.

[5] I. Daubechies, Ten Lectures on Wavelets, Society for Industrial and Applied Mathematics, Philadelphia, Pa, USA, 1992.

[6] W. Hardle, G. Kerkyacharian, D. Picard, and A. Tsybakov, Wavelets, Approximation and Statistical Applications, Springer, New York, NY, USA, 1997.

[7] A. B. Tsybakov, Introduction to Nonparametric Estimation, Springer, Berlin, Germany, 2009.

[8] R. DeVore, G. Kerkyacharian, D. Picard, and V. Temlyakov, "Approximation methods for supervised learning," Foundations of Computational Mathematics, vol. 6, no. 1, pp. 3-58, 2006. 


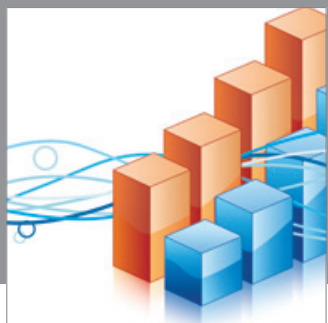

Advances in

Operations Research

mansans

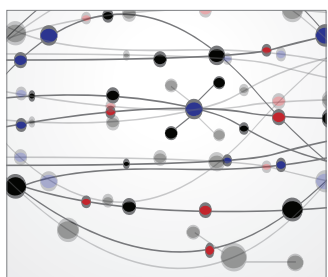

The Scientific World Journal
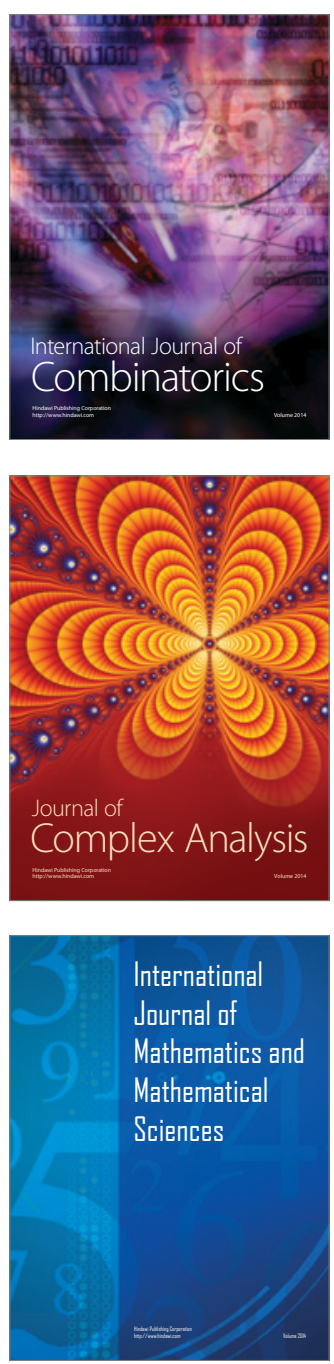
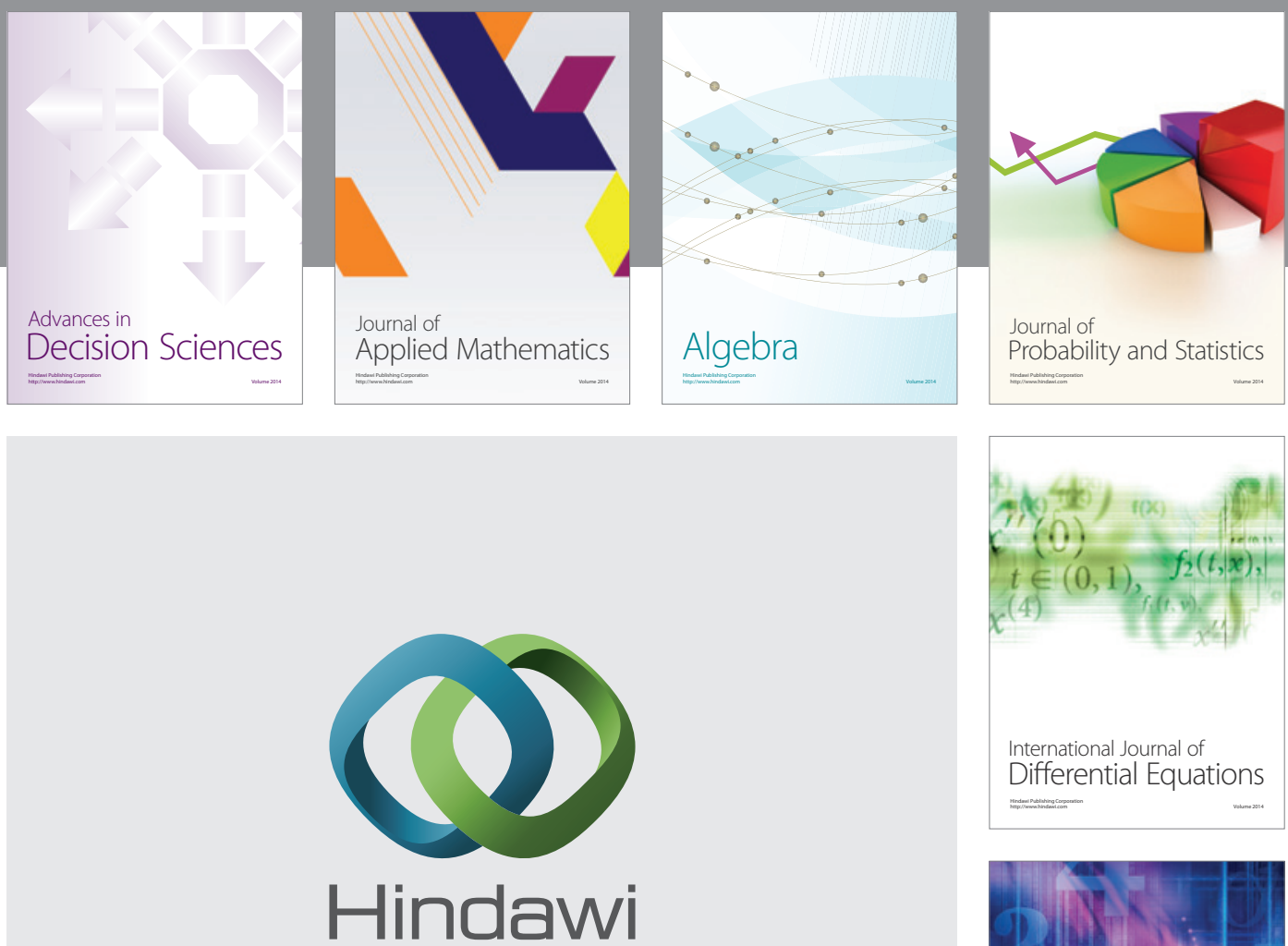

Submit your manuscripts at http://www.hindawi.com
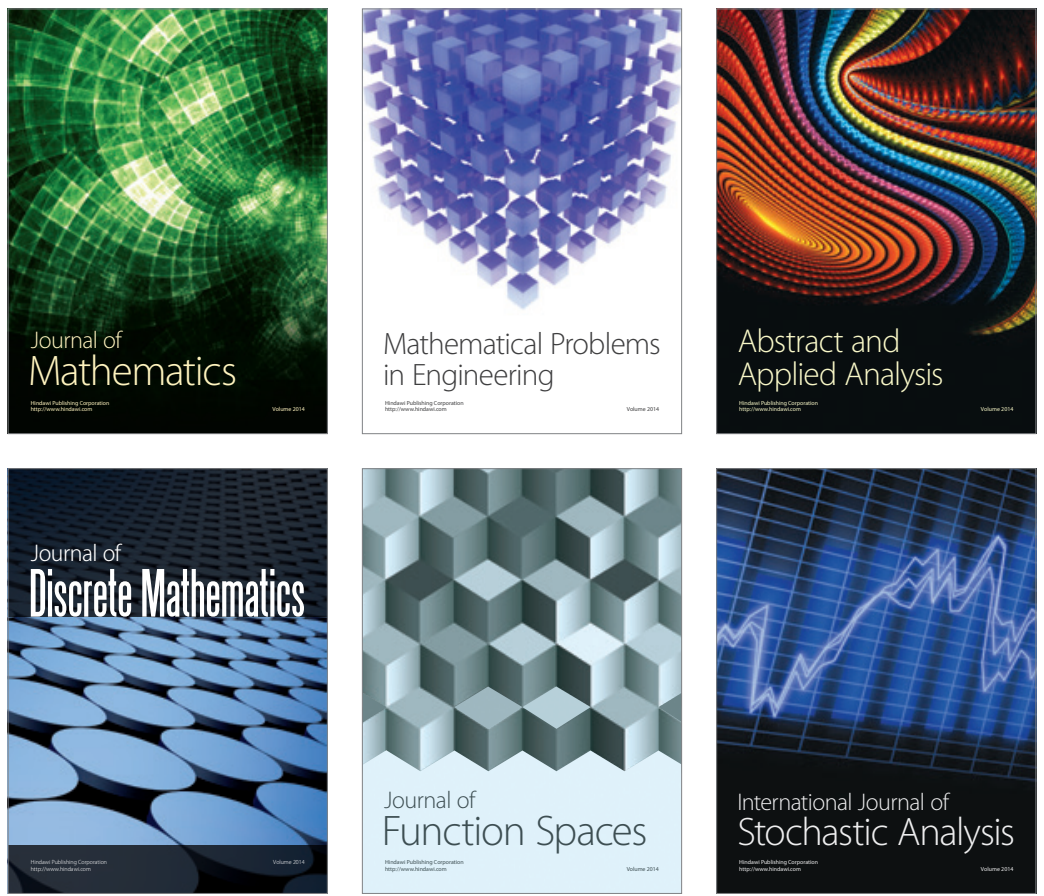

Journal of

Function Spaces

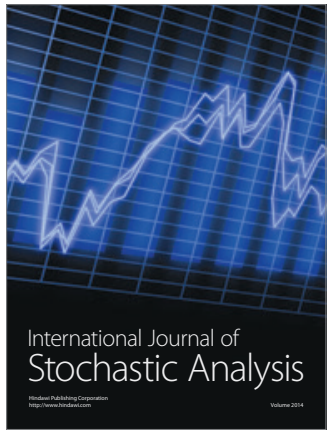

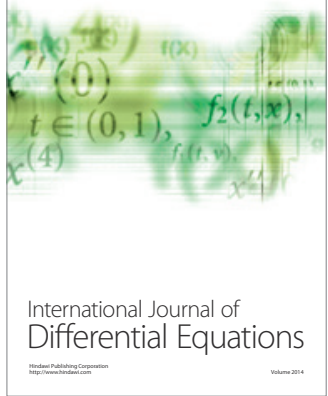
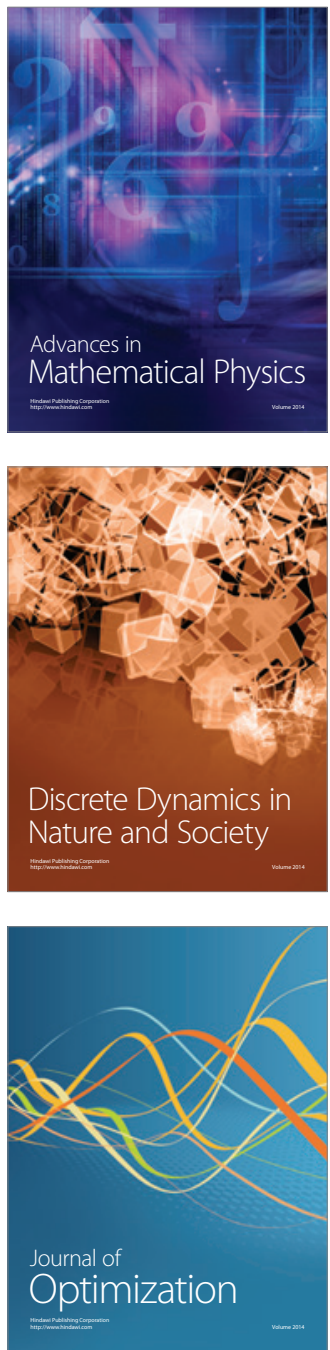